\title{
New guidelines unlikely to bring 'business as usual' back to dentistry
}

The British Dental Association has said new standard operating procedures for dentists in England are unlikely to represent a silver bullet for restoring patient access to services.

The new model places patients on two pathways, given the likelihood of them suffering from respiratory illness. It replaces restrictions that have been all but universal for most treatments and most patients since the first lockdown, which has radically reduced capacity across the service.

Those on the non-respiratory pathway can be managed in line with preCOVID-19 standard infection control precautions. Any placed on the respiratory pathway will remain subject to enhanced precautions, including maintaining 'fallow time' gaps of up to an hour between treatments. Screening to determine the pathway will take place both before attendance and on arrival the practice.

Dentist leaders anticipate a relatively high proportion of the population may find themselves on the respiratory pathway as the country heads into winter, given traditional patterns with seasonal flu and the common cold, even setting aside any surge in COVID-19 rates.

Over 35 million NHS dental appointments have been lost since the first lockdown, driven by ongoing restrictions. The service has not received a penny of additional investment from the Government's multi-billion-pound NHS 'catch up' programme to help bring down these backlogs, despite pressure

\section{'Dentist leaders anticipate a relatively high proportion of the population may find themselves on the respiratory pathway as the country heads into winter, given traditional patterns with seasonal flu and the common cold'}

BDA Chair Eddie Crouch said: 'We have long called for a roadmap to ease COVID19 restrictions. This approach is progress but will not represent a silver bullet for the tens of millions left without access to dental care.

'We cannot realistically expect a return to 'business as usual' as we head into winter given surging rates of respiratory disease.

'These new guidelines may help increase patient volumes but will barely make a dent into the unprecedented backlogs we now face. Without needed support from government we will continue to struggle to deliver for the patients that need us.'

from Healthwatch England and a growing number of MPs.

Unlike their colleagues in other UK nations dentists in England have received no capital funding to invest in ventilation systems that can allow for increased patient volumes while keeping to COVID19 restrictions.
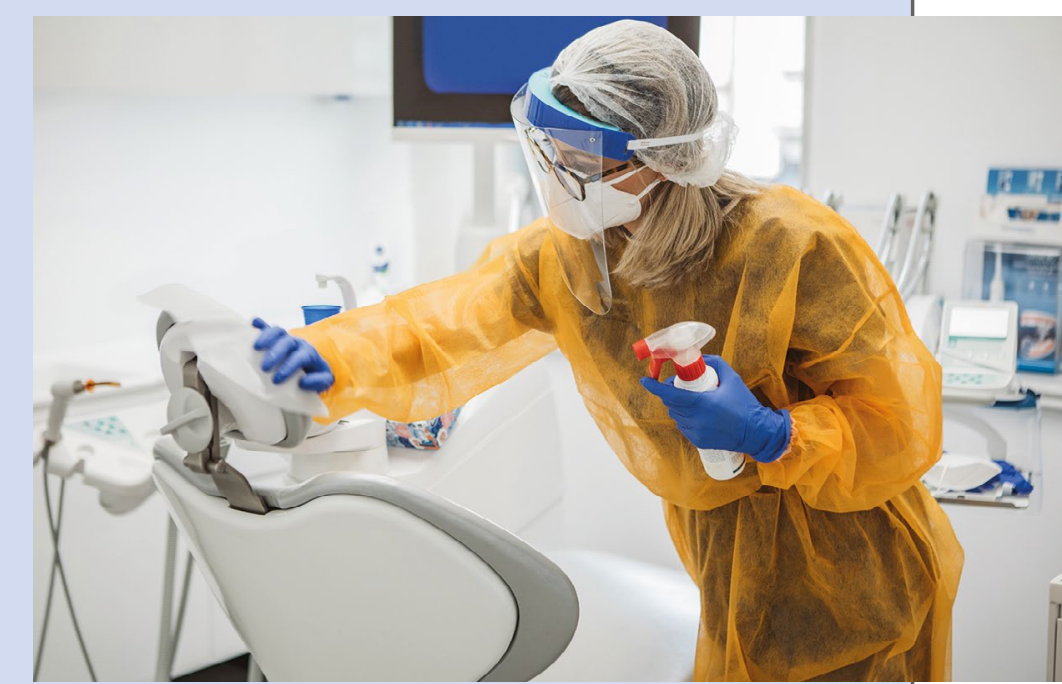

\section{BOS joins European consensus: 'DIY Orthodontics' is hazardous for patients}

The British Orthodontic Society is delighted to join a Europe wide consensus that sees 31 professional dental and orthodontic societies, associations, and institutions from 25 countries come together to endorse and fully support a Joint Declaration regarding the unacceptable and potentially unsafe remote treatment of malocclusions. This Declaration by EFOSA (European Federation of Orthodontic Specialists Associations) clearly states the basic requirements that must be met for any orthodontic treatment.

Dentists and orthodontists all over Europe are witnessing the increasing activities of start-up companies promoting and selling orthodontic treatment using aligners by post with great concern. This type of remote treatment is provided without either proper initial diagnosis or any form of regular clinical monitoring. These companies often present their services as affordable, fast, and safe, although they clearly do not meet required professional dental standards. Orthodontic treatment without proper initial diagnosis and regular clinical monitoring can cause severe risks to patients' health.

'The unanimity shown by European orthodontists on this subject makes it clear that orthodontics is more than just aligning the front teeth. It is about a holistic approach to care where the patient's best interests are at the heart of our treatments, Prof. Dr.
Christian Scherer, who coordinated the project for EFOSA, added. 'Every patient should make sure that the basic requirements formulated in the Joint Declaration are also observed in his or her treatment so that their treatment is practised safely'.

Anjli Patel, Director of External Relations for the British Orthodontic Society said: 'Orthodontic treatment without thorough clinical face-to-face examination of the patient, $\mathrm{x}$-ray imaging and regular clinical monitoring, is potentially hazardous to the patient's health. Any self-administered and remote treatment cannot be justified from a professional medical perspective and thus represents a serious violation of ethical, medical and dental standards.' 